\title{
Control Integrated Studies on High SPEed Permanent Magnetic GENERATORS SYSTEM
}

\author{
Jing. $\mathrm{Li}^{1}$, Xiaochen. Zhang ${ }^{1,2}$, He. Zhang ${ }^{1,4}$, Xiaoyan Huang ${ }^{3}$ and Gerada. Christopher ${ }^{1,4}$ \\ ${ }^{1}$ Department of Electrical and Electronic Engineering, University of Nottingham, Nottingham, NG7 2RD UK \\ ${ }^{2}$ School of Electrical Engineering, Beijing Jiaotong University, Beijing, 100044 China \\ ${ }^{3}$ College of Electrical Engineering, Zhejiang University, Hangzhou, Zhejiang, 310027 \\ ${ }^{4}$ IAMET, University of Nottingham, Ningbo, Zhejiang
}

The power converter and its control system has great impact on the behavior of the high speed permanent magnet generator system (HSPGS) due to the high operating frequency, and therefore should be investigated in the design and research on the HSPGS. This paper investigates a $3 \mathrm{~kW} \mathrm{80,000} \mathrm{rpm}$ HSPGS with the control system and evaluates machine performance. A study model composed of three dimensional finite element analyses and control system is proposed, and the magnetic density distribution in machine is investigated via FEA and power converter control directly coupled research. From the comparisons of simulation results of generator operating with or without control system, the effects of control system current time harmonics on the three dimensional flux distribution, the vector eddy current in rotor sleeve, and the iron loss of the machine are explored. By using the harmonics analyses, both the space flux harmonics and the voltage current time harmonics are obtained. The tested waveforms and voltage and current value of machine under different working conditions verify the calculated results. The obtained results could provide reference for system level design and investigation for HSPGS.

Index Terms - control, FEA, HSPGS, loss, harmonics

\section{INTRODUCTION}

$\mathrm{W}^{\mathrm{i}}$ ith advantages of low emissions, fuel flexibility, high efficiency and energy saving, micro turbines (MT) are one of the most important technologies for distributed generated systems (DGS)[1],[2]. With many advantages such as high power density, simple structure, and high efficiency, high speed permanent magnetic generator systems (HSPGS) attract more attentions from the world wide researchers and manufacturers.

The operation speed of HSPGS may be as high as tens of thousands revolution per minute, and the alternating frequency of the electromagnetic field could be as high as thousands of Hertz. Therefore the generated electrical power has to be transferred to the mains frequency or to DC via power converter, which makes the control system play an important role in the HSPGS. In such system, the time harmonics of armature current caused by the power conversion generates magnetic filed harmonics inside the motor, which affects generator performance directly and increases the eddy loss in rotor metal sleeve [3]. Therefore it is important to investigate the electromagnetic distribution in the generator under a certain power conversation with the integrated modelling of the power generation system.

A metal sleeve is often applied to rotor outer surface to retain the magnets and ensure the mechanical stability in a high speed generator application. The magnetic harmonics in air-gap, either caused by machine space structure or the controlled current time harmonic, would induce a complicate three dimensional distributed eddy current in rotor sleeve,

Manuscript received March 20, 2015. Corresponding author: Xiaochen. Zhang (e-mail: xchzhang@bjtu.edu.cn).

Digital Object Identifier inserted by IEEE which affects machine flux distribution and loss distribution [4]. Therefore it is beneficial and necessary to investigate the machine via three dimensional field calculations, especially for the machine with a cylindrical structure, in which the end windings have much more effects on space magnetic field distribution and therefore the machine performance [5].

Recently, many researchers devote to the performance studies of high speed machine, and some important research results have been achieved [6-8]. Most of these studies focus on the structure design [6], the thermal management [7], and optimizations [8], without considering the effects of the power converter control strategies. In this paper a $3 \mathrm{~kW}, 80,000 \mathrm{rpm}$ HSPGS is investigated together with power converter control system. The three dimensional flux distribution and machine output performance fed from power converter are investigated via a FEA and control strategy coupled modeling.

\section{THREE DIMENSIONAL MAGNETIC FIELD ANALYSES}

The machine studied in this paper is a HSPGS with a cylindrical structure used in micro turbine. The stator armature windings are designed as concentrated windings to reduce the machine total length and better thermal dissipation. Fig. 1 shows the prototype on the test rig and the structure.

The rotor is excited by the Permanent Magnets (PM) which is protected by a metal sleeve (titanium alloy). The magnets are made of $\mathrm{NdFeB35}$, the magnetic remanence of which is $1.1 \mathrm{~T}$, magnetic coercivity is $890 * 10^{3} \mathrm{~A} / \mathrm{m}$, and its limited working temperature is $180^{\circ} \mathrm{C}$. The main parameters of the prototype are shown in Table I.

Since a cylindrical structure is adopted, the effect caused by machine end windings can't be ignored, and a threedimensional whole region electromagnetic calculation model 


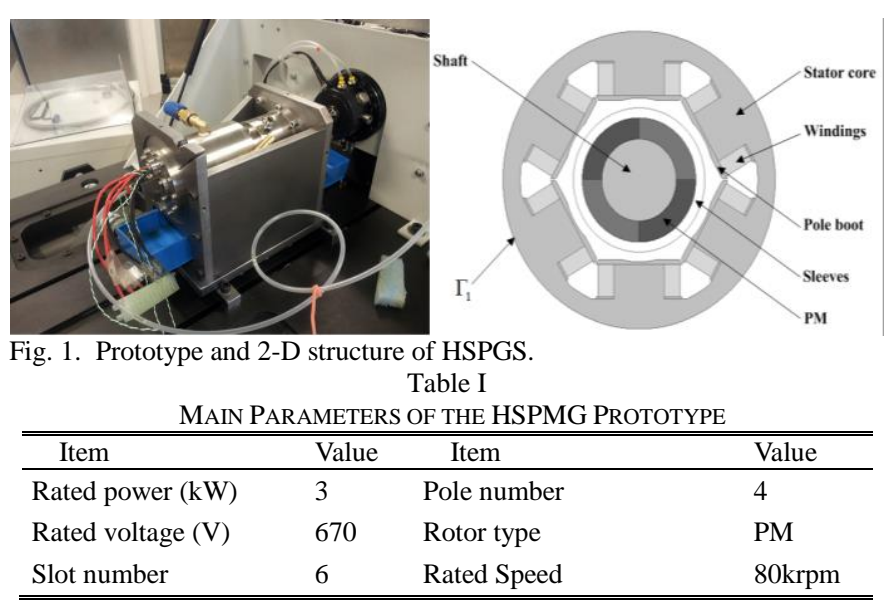

is established. The following assumptions are proposed.

(a) The stator windings is chosen to be stranded coils, and the outer diameter of the parallel wire is thin, thus the displacement current and Kelvin effect in stator core and windings are ignored in this calculation.

(b) Materials are isotropic, and the hysteresis effect of ferromagnetic materials is ignored.

(c) The influences of the temperature on material conductivity and permeability are ignored, and all the material characteristics are considered as that under the designed working temperature 80 Celsius degree.

The mathematical model of the transient magnetic field was established by the electric vector potential $\boldsymbol{T}$ and the scalar magnetic potential $\varphi$, and the equations are given by (1) and (2). That is in eddy current region

$$
\left\{\begin{array}{l}
\nabla \times \rho \nabla \times \boldsymbol{T}-\nabla \rho \nabla \cdot \boldsymbol{T}+\frac{\partial \mu(\boldsymbol{T}-\nabla \varphi)}{\partial t}+\frac{\partial \mu \boldsymbol{H}_{s}}{\partial t}=0, \\
\nabla \cdot \mu(\boldsymbol{T}-\nabla \varphi)=-\nabla \cdot \mu \boldsymbol{H}_{s}
\end{array}\right.
$$

and in the no eddy current region

$\nabla \cdot \mu \nabla \varphi=\nabla \cdot \mu \boldsymbol{H}_{s}$,

where

$$
\boldsymbol{H}_{s}=\frac{1}{4 \pi} \int_{\Omega} \frac{\boldsymbol{J}_{s} \times \boldsymbol{r}}{r^{3}} \mathrm{~d} \Omega
$$

Boundary conditions

$$
\left.\frac{\partial \varphi}{\partial n}\right|_{S_{1}}=0
$$

The initial conditions when $t=0$, i.e.,

$$
\left\{\begin{array}{l}
\left.\boldsymbol{T}\right|_{\Omega E}=\boldsymbol{T}_{0}(r, \theta, z) \\
\left.\varphi\right|_{\Omega}=\varphi_{0}(r, \theta, z)
\end{array}\right.
$$

where $\mu$ is the permeability(in henry per meter); $\boldsymbol{T}_{0}$ and $\varphi_{0}$ are the electric vector potential and the scalar magnetic potential at the initial time, respectively; $\rho$ is the resistivity (in ohm meters); $\boldsymbol{J}_{S}$ is the source current density in the windings (in ampere per square meter); $t$ is the time (in
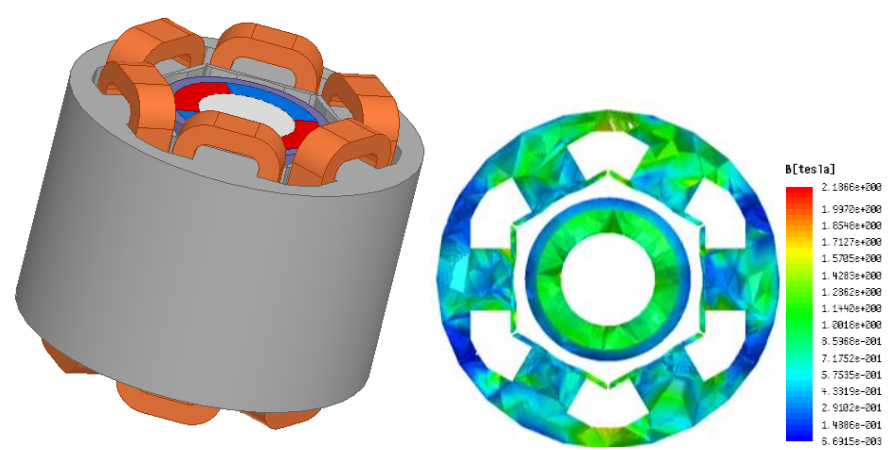

Fig. 2. The 3-D calculation model and magnetic density distribution.

seconds); $n$ is the normal vector of the surface; $S_{1}$ is the outer surface of the calculation region; $\Omega$ is the calculation region; $\Omega_{E}$ is the eddy current region; $r, \theta$, and $z$ are the radial, circumferential, and axial directions, respectively.

Fig. 2 shows the three dimensional whole region electromagnetic field calculation model, in which the end windings are established according to the actuary size. The analysis model of the HSPGS is meshed with tetrahedron elements. By using the time-stepping finite-element method, the electric vector potential and the scalar magnetic potential of every node can be calculated, and then, the flux density distribution inside machine can be determined. Fig.2 also shows the flux density distribution in a cross section of the machine, which is obtained from the machine operating with a resistance loading without the control system.

The eddy current losses occur in the solid sleeve caused by the air-gap flux density harmonics. The eddy current losses of the HSPGS fed from PWM power converter could be very large and become dominate Therefore, it is very critical to determine the eddy current losses accurately. Such eddy current losses in components can be calculated by using the obtained induced eddy current. Thus, the three-dimensional rotor eddy loss in sleeve can be determined by summing full volume current loss. The rotor eddy current loss are calculated by

$$
P_{e}=\frac{1}{\mathrm{Te}} \int_{\mathrm{Te} \text { vol }} \int_{e} J_{e}^{2} \sigma_{r}^{-1} \mathrm{~d} V \mathrm{~d} t
$$

where, $P_{e}$ is rotor eddy current (in watts), $J_{e}$ is eddy current density in element $e$ (in ampere per square meter), $\sigma_{r}$ is rotor sleeve conductivity (in siemens per meter/m), Te is the cycle of time, and vol is the region of eddy current losses.

\section{INFLUENCE OF POWER CONVERTER SYSTEM}

To investigate the influence of power converter system on machine losses and HSPGS performance, a direct coupled system level model is established, based on the proposed FEA three dimensional model. Then, the three dimensional electromagnetic field is transient-transient and step-step cosimulated with the power converter system [6], and the interactions between the generator and the power converter control system can be investigated 


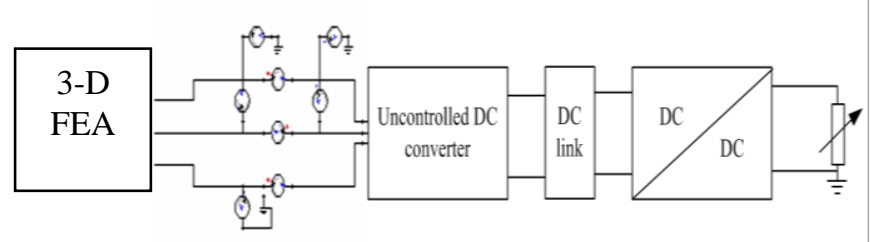

Fig. 3 Control integrated analysis model.

Fig. 3 shows the system level analysis model for the HSPGS in this paper, in which the three dimensional FEA is connect to a power converter system, which includes the uncontrolled rectifier, the DC-DC converter, the DC link filter, and the equivalent load resistance. The interaction between the high speed machine and the control system are modelled via the time varying current and terminal voltage in the co-simulation circuit. The alternating frequency of flux inside machine is very high, the switching frequency of the device is $20 \mathrm{k} \mathrm{Hz}$, and the gain of DC-DC converter is 0.3 , and the modulation radio is 15 . The equivalent load resistance is $0.32 \mathrm{ohm}$.

Fig.4 shows the comparisons of the phase current and line to line voltage of the high speed generator operating under $80 \mathrm{k}$ rpm and $2.8 \mathrm{~kW}$ output between the experimental results and simulated curves. In the view of time domain, the simulation results show good agreements with measured ones, The experimental results have much more high frequency harmonics than the simulation due to the operation of the rectifier and DC-DC converter. As the uncontrolled rectifier is connected to machine directly without inductors and the rectifier input frequency is as high as $2.67, \mathrm{kHz}$, the line to line voltage is three-level square wave with voltage-zeros. There is a small dip in current half cycle, resulting from the armature reaction and machine slot structure,. The calculated current amplitude is about $6 \%$ higher than the test value, and there is $3 \%$ difference in voltage respectively.

In order to investigate the effects caused by the connected power conversion system (PCs), the comparison tests have been taken out for high speed permanent generator with or without PCs, respectively. Table. II shows the variations of machine output terminal voltage and current with and without PCs. When with the PCs the $238 \mathrm{~V}$ AC line to line voltage is converted to $16.2 \mathrm{~V} \mathrm{DC}$ voltage, and the $2.4 \mathrm{~A}$ phase current AC current is converted to $49.5 \mathrm{~A}$ DC current while generator operating under $70 \mathrm{k} \mathrm{rpm}$. In this test, the input power of the generator are both 0.185 N.m, and it was measured that the efficiency of machine reduces from $71.8 \%$ to $71.4 \%$ after the implementation of PCs, and the efficiency of HGPGS is only $59.1 \%$. Whereas for operating under speed of $80 \mathrm{k} \mathrm{rpm}$, the system efficiency is reduced about $11 \%$ when has an output power of $2.8 \mathrm{~kW}$.

Also the operations of HSPGS under different load conditions are co-simulated, by using the three dimensional FEA and integrated analysis model, respectively. The calculated results are list in Table. II. Comparing with the measured data, the largest difference is less than 3\%, appeared at the data of DC voltage operating under $80 \mathrm{k} \mathrm{rpm}$ with PCs. In different cases the calculated results are consistent to the tested data, and the accuracy of the proposed FEA model and integrated analysis model are verified.

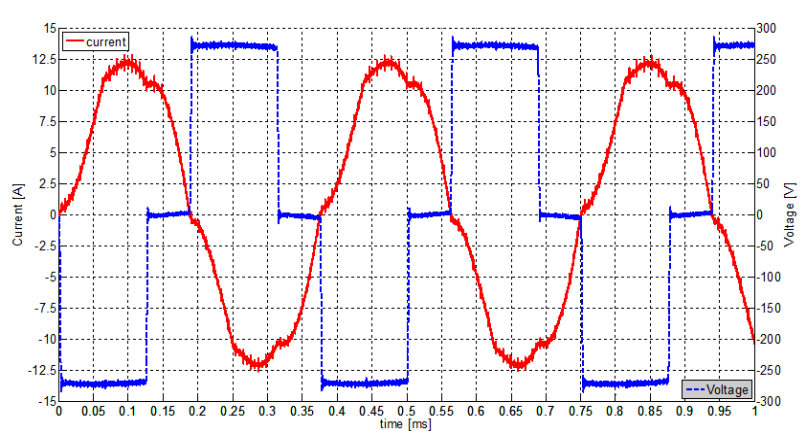

a) measured

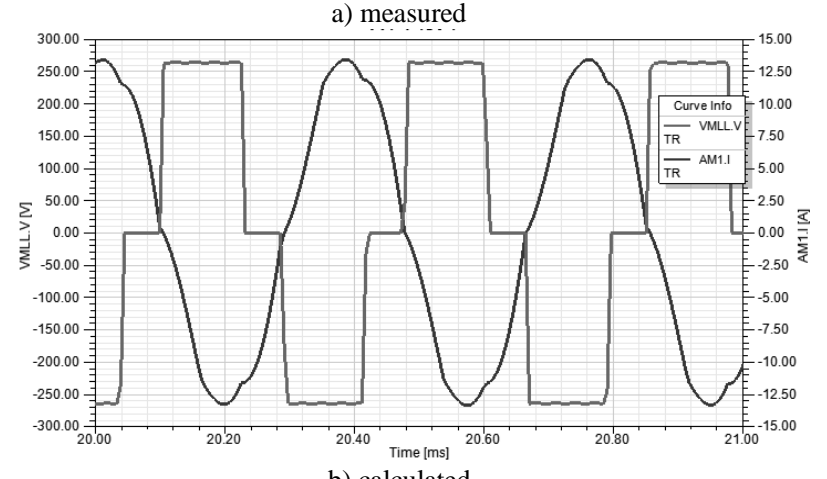

b) calculated

Fig. 4. Comparisons of the measured and calculated voltage and current.

TABLE II

COMPARISON OF CALCUlATED RESUltS WITH TEST DATA OF HSPGS WITH OR WITHOUT POWER CONVERTER

\begin{tabular}{c|clccc}
\hline \hline \multicolumn{2}{l}{ Speed (krpm) } & & measured calculated & error \\
\hline \multirow{3}{*}{\begin{tabular}{c|ccc} 
Without \\
PCs
\end{tabular}} & \multirow{2}{*}{70} & Line-Line Voltage (V) & 238.2 & 235.3 & $-1.22 \%$ \\
& \multirow{2}{*}{80} & Phase Current (A) & 2.4 & 2.36 & $-1.67 \%$ \\
\cline { 3 - 6 } & & Line-Line Voltage (V) & 242.7 & 237.5 & $-2.14 \%$ \\
& \multirow{2}{*}{70} & Phase Current (A) & 7.2 & 7.02 & $-2.50 \%$ \\
\hline \multirow{3}{*}{ With } & & DC Coltage (V) & 16.2 & 15.8 & $-2.47 \%$ \\
PCs & \multirow{2}{*}{80} & DC Voltage (V) & 29.5 & 28.7 & $-2.71 \%$ \\
& & DC Current (A) & 93.5 & 91.1 & $-2.57 \%$ \\
\hline \hline
\end{tabular}

Fig. 5 shows the induced eddy current density in the generator rotor sleeve with or without connecting to PCs.. The electronic device in the control system would cause high order current harmonics in machines windings, which increase the space magnetic field harmonics in machine air-gap, notly. These flux harmonics promote the generation of eddy current in rotor sleeve increase observably. From the eddy current vector distributions, it can be seen that, when the generator connected to a PCs, the eddy current in rotor sleeve distributed more centralized, and the maximum induced eddy current increase to $3.2910^{\wedge} 6$ to from $2.5010^{\wedge} 6$ (in Amperes per cubic meters). Based on the method introduced in [6], the eddy loss in rotor increased more than 3 times, that is from $6.24 \mathrm{~W}$ atts to 27.18 Watts, which would take much press for heat transmission in rotor side, and a better control scheme may need to reduce such eddy loss.

Besides the eddy current in rotor sleeve, the flux density in stator also affected by the PCs current harmonics, just as shown in Fig.6, the variations of partial magnetic density distribution before and after the connection of PCs. Under the operating with PCs, the maximum magnetic density up to 2.11 Tesla, and the saturation region also becomes larger. The total stator iron loss increase to 180 Watts from 113 Watts when the PCs connected, under operating of $80,000 \mathrm{rpm}, 2.8 \mathrm{~kW}$. 


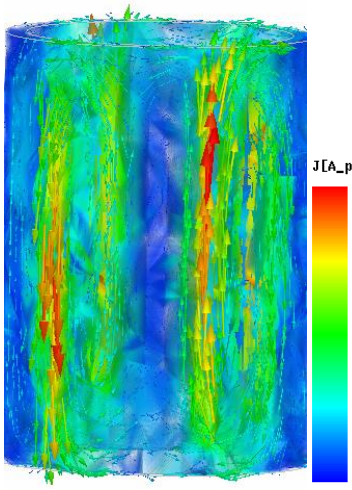

a) without PCs

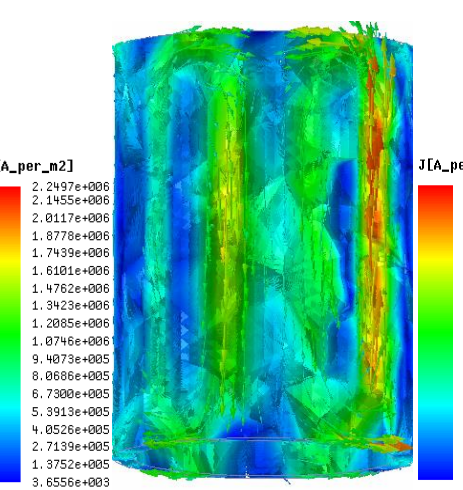

b) with PCs
Fig. 5. Comparisons of eddy current density distribution in rotor sleeve.

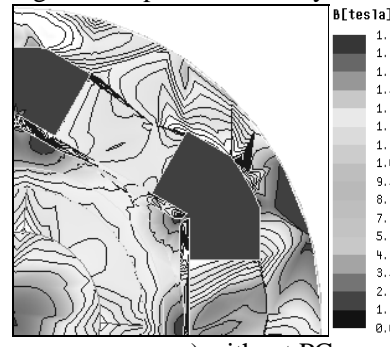

a) without PCs

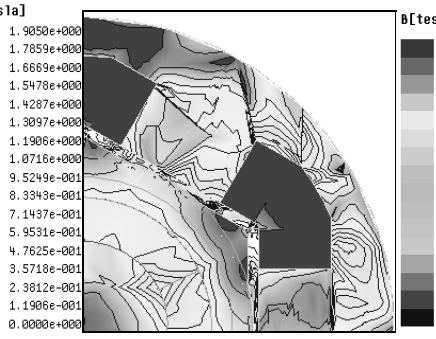

b)with PCs
Fig. 6. Comparisons of magnetic density distribution.

Fig.7 presents the harmonic analyses on the HSPGS. Comparing with HSPGS without PCs, the harmonics of machine terminal voltage with power converters increase obviously, which are mainly the $6 \mathrm{k} \pm 1(\mathrm{k}=1,2,3 \ldots$.$) orders of$ the fundamental frequency due to the operation of the uncontrolled rectifier, as shown in Fig.7 a) and b). Meanwhile, the space harmonics in air-gap also changed significantly, and the harmonics contents increase dramatically, as shown in Fig.7 c) d) and e). Therefore, the enhanced control strategy of the power converter system needs to be investigated in the future to reduce the harmonic contents in the machine phase currents.

\section{CONCLUSION}

In this paper, a three-dimensional FEA analysis and power converter coupled optimization method is applied to a high speed generator with the main objective of reducing the eddy current loss in sleeve and minimizing harmonics. By comparing with the experimental results, the proposed integrated study model is verified with high accuracy. The eddy current losses in the sleeve increases dramatically after connecting to the power converter, sufficient considerations should be taken for designing high speed generator connected with power converter. As future work, measures of reducing the eddy current losses in the metal sleeve should be further investigated.

\section{ACKNOWLEDGMENT}

This work is supported by the National Natural Science Foundation of China under Grant 51407006.

\section{REFERENCES}

[1] O. Aglen. "A high speed generator for microturbines". IEEE International Conference on Electric Machines and Drives, 2001, 1-5.

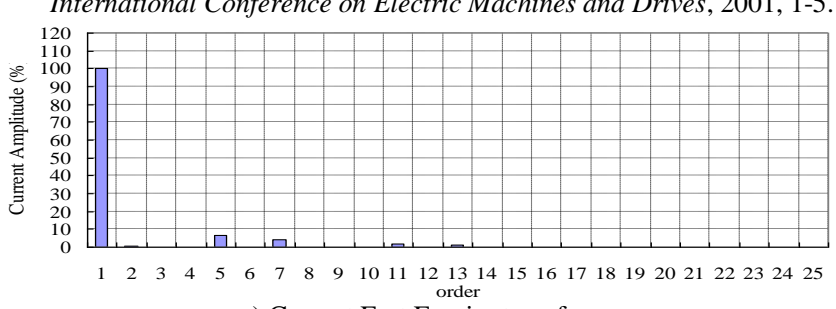

a) Current Fast Fourier transform

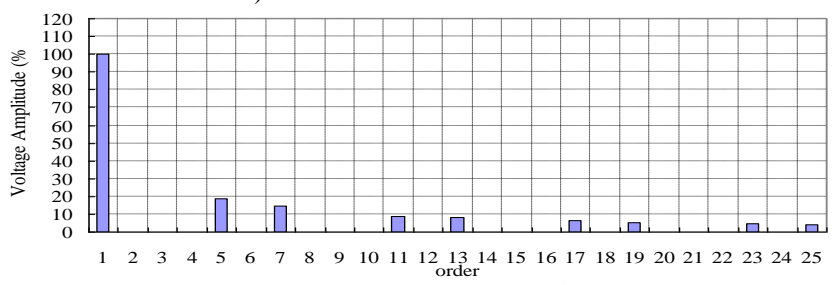

b) voltage Fast Fourier transform

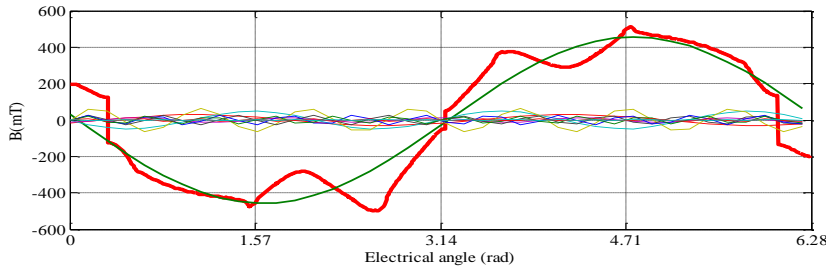

c) flux density and its harmonics in machine with out PCs

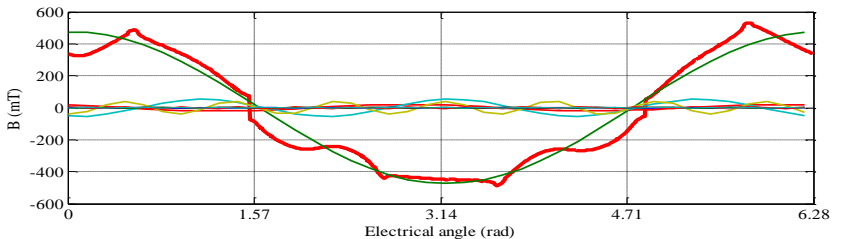

d) flux density and its harmonics in machine with PCs

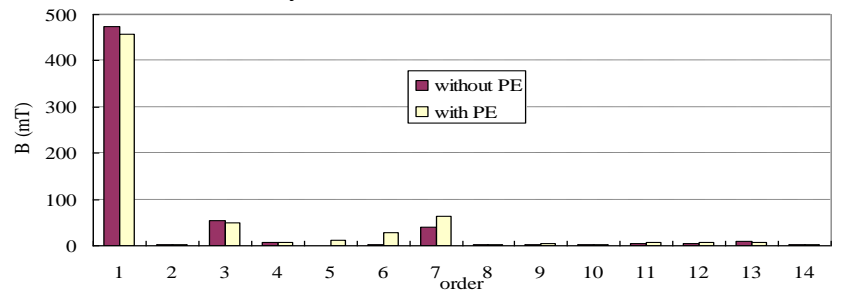

e) flux density harmonic

Fig. 7. Harmonics analyses for HSPGS.

[2] H. Do-Kwan, W. Byung-Chul, L. Ji-Young, K. Dae-Hyun. "Ultra High Speed Motor Supported by Air Foil Bearings for Air Blower Cooling Fuel Cells". IEEE Trans. on Magn., 2012, Vol. 48, No. 2, pp. 871-874.

[3] S. Niu, S. L. Ho, W. N. Fu, Z. Jian Guo. "Eddy Current Reduction in High-Speed Machines and Eddy Current Loss Analysis with Multi slice Time-Stepping Finite-Element Method". IEEE Trans. Magn., 2012, Vol. 48, No. 2, pp. 1007-1010.

[4] D. Gerada, A. Mebarki, N.L. Brown, et al. "Design Aspects of HighSpeed High-Power-Density Laminated-Rotor Induction Machines", IEEE Trans. Ind. Electron., vol. 58, no. 9, pp. 4039-4047, Sep. 2011.

[5] M. Mirzaei, A. Binder, B. Funieru, M. Susic. "Analytical Calculations of Induced Eddy Currents Losses in the Magnets of Surface Mounted PM Machines with Consideration of Circumferential and Axial Segmentation Effects". IEEE Trans. Magn., 2012, Vol. 48, No. 12, pp. 4831-4841.

[6] M. Jagiela, T. Garbiec, M. Kowol. "Design of High-Speed Hybrid Hysteresis Motor Rotor Using Finite Element Model and Decision Process". IEEE Trans. Magn., 2014, Vol. 50, No. 2, \# 7021304.

[7] X. Zhang, W. Li, B. Kou, et al. "Electro-thermal combined optimization on notch in air cooled High Speed Permanent Magnetic Generator", IEEE Trans. Magn. 2015, Vol. 51, No. 1, \# 8200210 . 
[8] O. Maloberti, R. Figueredo, C. Marchand, et.al. "3-D-2-D Dynamic Magnetic Modeling of an Axial Flux Permanent Magnet Motor With Soft Magnetic Composites for Hybrid Electric Vehicles". IEEE Trans. Magn., 2014, Vol. 50, No. 6, \# 8201511. 